\title{
Los analgésicos tópicos son efectivos para aliviar el dolor
}

Quantitative systematic review of topically applied non-steroideal anti-inflammatory drugs.

Moore RA, Tramer MR, Carroll D, et al. BMJ 1998; 316:333-338

\section{Objetivo}

Revisar la efectividad y seguridad de los antiinflamatorios no esteroides (AINE) tópicos en pacientes con dolor agudo y crónico.

\section{Fuente y selección de datos}

Selección de estudios randomizados y controlados de AINE tópicos, cuyo principal resultado a evaluar fuera dolor.

Se utilizó para la búsqueda la base de datos de Medline (1966-1996), Embase (1981-1996) y el Oxford Pain Relief (1950-1994).

Se solicitó información de estudios publicados y no publicados a doce empresas farmacéuticas del Reino Unido fabricantes de AINE tópicos.

\section{Resultados}

Se dividieron en dos condiciones:

a) Dolor aqudo: Se encontraron 40 estudios randomizados de los cuales 37 cumplieron los criterios para ser incluidos. De ellos, 27 estudios demostraron superioridad de los AINES tópicos respecto del placebo. Se encontró un beneficio relativo combinado de 1.7 (IC 95\% 1.5-1.9) y un número necesario para tratar (NNT) de 3.9 (IC 95\% 3.4-4.4). Tres estudios compararon los tratamientos de AINE tópico y AINE por vía oral, sin detectar diferencias.

Datos individuales sobre cada droga, que incluían por lo menos tres estudios randomizados y controlados, mostraron que el ketoprofeno, felbinac, ibuprofeno y piroxicam fueron significativamente superiores al placebo pero la indometacina y el benzidamina no mostraron diferencias.

b) Dolor crónico: Doce estudios cumplieron los criterios establecidos para ser evaluados, en siete los AINES tópicos fueron superiores al placebo. El beneficio relativo combinado fue de 2 (IC 95\% 1.5-2.7) y el NNT de 3.1 (IC 95\% 2.7-3.8).

Dos estudios que compararon AINE local vs. AINE por vía oral no mostraron diferencias.

\section{Conclusiones}

Los resultados demostraron que los AINES tópicos fueron significativamente más efectivos que el placebo para aliviar el dolor en condiciones agudas y crónicas.

Ningún estudio evidenció mayor efectividad de los AINES orales respecto de los administrados por vía tópica. Los efectos adversos de los AINES tópicos fueron iguales a los del placebo.

\section{COMENTARIO}

Esta revisión sistemática tuvo como objetivo evaluar la efectividad y la seguridad de los antiinflamatorios tópicos, comparándolas contra placebo, entre sí y con drogas antiinflamatorias no esteroideas administradas por vía oral.

Los pocos estudios publicados hasta ahora estaban limitados a un bajo número de pacientes, por lo cual fue necesaria una revisión sistemática que pudiera combinar los resultados. Se empleó una amplia estrategia de búsqueda y una evaluación de la calidad de la metodología de los estudios con un score del 1 al 5. Esta tenía en cuenta la calidad de la randomización, evaluaba si los estudios eran ciegos, los criterios de selección y la pérdida de pacientes. Mas del 75\% de los estudios evaluados tuvieron un puntaje mayor a tres.

De acuerdo a esta revisión sistemática de estudios controlados y randomizados, uno de cada tres pacientes que utilicen drogas AINES tópicas para dolores agudos o crónicos tendrá resultados favorables. Este efecto puede ser comparable al de los AINE por vía oral cuando se comparan con placebo en dolor leve a moderado ya que el beneficio absoluto en el alivio del dolor es similar. Los preparados tópicos tienen iguales efectos adversos que los obtenidos con el tratamiento placebo, sin el riesgo de hemorragia digestiva, que para el uso crónico de AINE por vía oral es del 1,3 al 1,6\% anual ${ }^{1}$. Los autores reportan que, si bien los estudios que compararon AINE tópicos con AINE administrados por vía oral no encontraron diferencias, el escaso número de participantes limitaba el poder de los mismos para responder esta pregunta.

Un problema que puede señalarse es la presencia del sesgo de publicación. Esto significa que habitualmente se publican los estudios en que los resultados son positivos. Sin embargo, para solucionar este problema los autores realizaron esfuerzos para conseguir datos de todos los estudios, inclusive aquellos no publicados.

Los AINE tópicos estuvieron disponibles comercialmente mucho tiempo antes de que existiera alguna evidencia sobre su utilidad, recién ahora se sabe de su eficacia y escasos efectos adversos. Su utilización resulta entonces ideal en aquellos pacientes con efectos adversos por AINE por vía oral o riesgo de hemorragia digestiva alta ${ }^{2}$.

\section{Dr. Martín Boccardo}

Unidad de Medicina Familiar y Preventiva. Hospital Italiano de Buenos Aires. 